\title{
Application of Heating Type Micro-Assembly Device in Two-Photon Micromachining
}

\author{
Jintao XIA, Afei DING, Pan WANG, Hang WANG, Yinwei GU, \\ Weidong $\mathrm{TAO}^{*}$, and Gang WANG ${ }^{*}$
}

School of Physical Science and Technology, Ningbo University, Ningbo 315211, China

*Corresponding authors: Weidong TAO and Gang WANG E-mails: taoweidong@nbu.edu.cn; gangwang@nbu.edu.cn

\begin{abstract}
The development of micro-fabrication and micro-assembly technology is indispensable for the future manufacturing of miniaturized, functional, and integrated devices. This paper proposes a planar micro-assembly technology to make the assembly of micro-objects easier. Firstly, delicate three-dimensional (3D) structures were fabricated on glass and silicon slice substrates using femtosecond laser two-photon polymerization (2PP). Secondly, transparent fluorescent scintillation ceramic powder, referred to as fluorescent powder, was assembled using a laboratory-made 3D moving heating micro-operator into a microstructure on a glass substrate, and this device is used to assemble the graphene powder into the microstructure on the silicon slice substrate. The fluorescence spectra and Raman spectra characterizations of the fluorescent powder and graphene powder in the microstructure were carried out by using excitation light at $405 \mathrm{~nm}$ and $532 \mathrm{~nm}$, respectively. According to the above results, it can be concluded that the powder properties of the fluorescent powder and graphene powder assembled into the microstructure were not changed. The experimental device could not only assemble many micron-sized powder materials into hollow microstructures of arbitrary shape but also joined microstructures with different materials and characteristics to form a complex hybrid microstructure system.
\end{abstract}

Keywords: Two-photon polymerization; micro-assembly; fluorescence; graphene

Citation: Jintao XIA, Afei DING, Pan WANG, Hang WANG, Yinwei GU, Weidong TAO, et al., "Application of Heating Type Micro-Assembly Device in Two-Photon Micromachining," Photonic Sensors, 2021, 11(3): 362-370.

\section{Introduction}

With the rapid development of the micro-nano technology, the trend of product miniaturization is increasingly apparent. More and more precise micro-devices were manufactured and applied in various advanced fields, such as aerospace [1], automobile manufacturing [2], genetic engineering [3], and life sciences [4]. At present, the importance of micro-assembly and the micromanipulation technology has become increasingly prominent.
Because micro-assembly is mainly aimed at micron-level devices, which are light in weight, low in energy consumption, and low in inertia, the assembly is difficult, during the assembly process. Therefore, for the precision type, the assembly of micro devices has gradually become the focus of attention in the industry [5]. For example, Köhler et al. [6] used a holographic optical device to capture, move, and assemble microstructures of different structures in a non-contact manner by using their optical forces to form complex hybrid 
microstructures. Villangca et al. [7] created a new type of visual robot by using light-induced convection to assemble silicon slice and polystyrene beads into the body of a robot. Frazier et al. [8] fabricated micro-gears and micro-shafts based on the polyimide lithography technology. The copper micro-gears and micro-shafts were made of electroformed copper and assembled by using the post-assembly technology, and the gears could rotate freely around the shaft. This article focuses on a self-made three-dimensional (3D) mobile heating micro-manipulation device that can perform highly efficient and flexible operations on micron-sized objects. Due to that the femtosecond laser two-photon polymerization (2PP) technology has the true 3D manufacturing capability, high spatial resolution, and fineness of up to the micro-nano level, it is an ideal tool for the preparation of $3 \mathrm{D}$ micro-nano structures [9-11]. Therefore, the paper uses the 2PP technology to create the micro-scale functional integrated microstructure. In this paper, the $3 \mathrm{D}$ mobile heating micro-manipulation device was used to capture, fix, and assemble the fluorescent powder into the microstructure by micro-operation technology. At the same time, it was experimentally confirmed that the technology could be used to assemble the graphene powder into the microstructure. This new type of micromanipulator provided a material assembly mode that might provide a new solution for the applications such as micro-device assembly, bioluminescent labeling, and medical drug delivery [12].

\section{Materials and methods}

\subsection{Principle of two-photon polymerization}

In the process of initiating two-photon absorption (2PA), a very high energy density was required at the focal point. The peak power of the femtosecond laser was much higher than that of the continuous laser and the nanosecond pulsed laser. Because the pulse width of the femtosecond laser leaded to extremely high photon density in the focal region, it just met the energy density requirement of 2PA [13]. Compared with the traditional single-photon polymerization (1PP), one of the main advantages of 2PP is its high spatial resolution and exceptional 3D engraving ability inside the photoresist [14-16]. The femtosecond laser emitted infrared or near-infrared light, and due to insufficient energy inside the photoresist, 1PP could not be induced. When two infrared photons met at the focal point, the power reached the required value for the polymerization, so 2PP could only be activated at the focus. Therefore, the femtosecond laser beam could penetrate the photoresist and induced internal polymerization of the liquid at the focus without the need to polymerize the photoresist along its path [17]. Figures 1(a) and 1(b) show the difference between $2 \mathrm{PP}$ and $1 \mathrm{PP}$, respectively. $2 \mathrm{PP}$ only causes polymerization at the focal point of the femtosecond laser beam, scanning a "LASER" pattern along the movement trajectory, while 1PP initiates polymerization throughout the laser path, scanning a "LASER" pattern. Due to the energy threshold requirement of $2 \mathrm{PP}$, nanoscale resolution could be achieved by controlling the laser pulse energy and the writing speed.

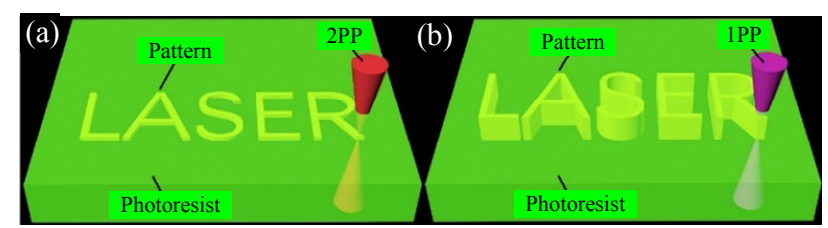

Fig. 1 Schematic diagram of 2PP: (a) two-photon direct write "LASER" pattern and (b) single-photon direct write "LASER" pattern.

\subsection{Femtosecond laser micromachining device diagram}

The glass slide and silicon slice sheets were cut into $5 \times 5 \mathrm{~mm}^{2}$, glass slide and silicon slice sheets with acetone solution ultrasound were treated for $10 \mathrm{~min}$, the oil stain on the substrates surface was cleaned and ultrasonically cleaned again with alcohol for $10 \mathrm{~min}$, and the residual stains were washed twice. The glass flakes and the silicon slice flakes were ultrasonically cleaned twice with 
deionized water for $10 \mathrm{~min}$, the residual acetone and alcohol on the surface were then completely cleaned, and finally they were dried with nitrogen to obtain a relatively clean substrate. The substrate was placed in an ultraviolet (UV) light cleaning machine (BZS250GF-TS) for light cleaning, then the photon energy could form a highly polar functional group $(-\mathrm{OH},-\mathrm{CHO}$, and $-\mathrm{COOH})$ on the surface of the substrate, and the activated functional group had good adhesion force and bonding properties. These functional groups were bonded with negative photoresist to form new chemical bonds, so that the microstructure and substrate after preparation had stronger adhesion and were not easy to fall off [18]. The preparation of the sample for the micromachining experiment is shown in Fig. 2. The cleaned substrate was mounted on a glass slide, and a spacer of $150-\mu \mathrm{m}$ height was placed on both ends of the substrate with an interval of about $3 \mathrm{~mm}$. A drop of negative photoresist was placed in the center of the substrate with a pipette, and the cover glass was slowly released at one end of the spacer to uniformly spread the photoresist on the substrate to prevent bubbles from being generated inside the photoresist liquid. The femtosecond laser was focused through the lens numerical aperture (NA=0.65), and was directly focused on the inside of the photoresist through the cover glass. The cover glass was supported by the gasket so that the femtosecond laser could be scanned inside the photoresist with a thickness of $150 \mu \mathrm{m}$.

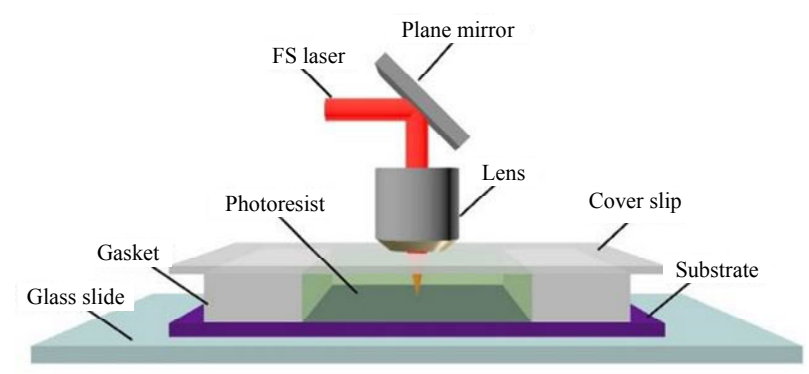

Fig. 2 Micro-machining sample device diagram.

\subsection{Fabrication of microstructure}

The 3D drawing software was used to draw a 3D "arch bridge" shaped hollow structure, as shown in Fig. 3(a). The length, height, and width of the microstructure were all $120 \mu \mathrm{m}$, and there was a small circular hole with a diameter of $30 \mu \mathrm{m}$ on the top. The laser could be directly irradiated inside of the microstructure. The "arch bridge" shaped structure was saved as the stereolithography (STL) format and imported into the uFAB supporting control software of micromachining station and supplied by Newport Company, and the software automatically sliced the 3D pattern from bottom to top along the $Z$-axis direction. The prepared sample was placed on a micromachining stage and fixed, and a $780 \mathrm{~nm}$ wavelength femtosecond laser (Coherent 2; Mira-900f, wavelength $780 \mathrm{~nm}$ ) was excited by a mode-locked Ti: Sapphire laser was used as a light source. The laser power was adjusted to $450 \mu \mathrm{W}$, and the scan speed was $50 \mu \mathrm{m} / \mathrm{s}$. The laser focus was from the bottom to the top, and the 3D pattern was written on the substrate by layer-by-layer scanning around the contour of the $3 \mathrm{D}$ pattern slice. The pattern produced by $2 \mathrm{PP}$ was immersed in anhydrous ethanol for $10 \mathrm{~min}$, so that the uncured photoresist was completely dissolved in absolute ethanol, and the photoreactive region (the curing zone) was retained. Finally, a cleaned 3D

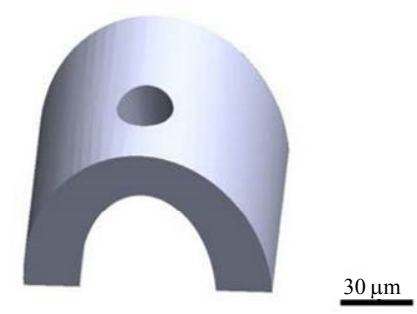

(a)

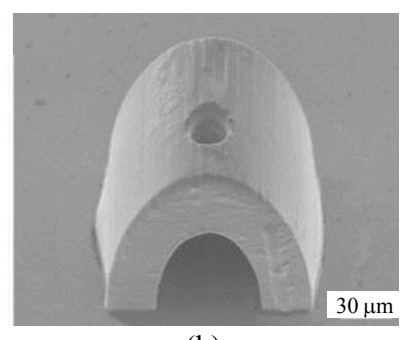

(b)

Fig. 3 Preparation of "arch bridge" shaped microstructures: (a) 3D design of microstructures and (b) SEM images of 40-fold magnifications. 
"arch bridge" shaped structure was obtained. In order to make the microstructure firmer, it was exposed to UV light for about $2 \mathrm{~min}$, so that the uncured photoresist inside the microstructures was completely cured. Figures 3(b) show scanning electron microscope (SEM) images of the "arch bridge" shaped structure. The pattern after polymerization is basically the same as the design drawing, reflecting the advantages of femtosecond laser fine processing.

\subsection{D moving heating device}

How to accurately assemble tiny objects into the microstructure has always been a difficult problem. In this experiment, the self-made 3D mobile heating micro-operation platform was used, and the movement precision could reach $1 \mu \mathrm{m}$, as shown in Fig. 4(a). The 3D moving platform was composed of three one-dimensional movement platforms. The heating device consisted of a heating probe and a tungsten steel probe (WG-38-10.0). The tip of the tungsten steel probe had a diameter of $20 \mu \mathrm{m}$, and the structure diagram is shown in Fig. 4(b). The heating device was fixed on the moving platform, and the probe tip was controlled to move on three planes of $X, Y$, and $Z$ by adjusting the upper and lower, front and rear, and left and right knobs of the 3D moving platform. First, a small amount of fluorescent powder was picked up by a capillary tube with a diameter of $500 \mu \mathrm{m}$, and placed in the center of the glass slide, then the slide was fixed on the stage of transmission microscope. Second, by adjusting the three-dimensional moving platform, the tip of the needle touched the fluorescent powder in the center of the slide. Finally, by rotating the front and rear knobs of the platform, the tip of the needle went deep into the fluorescent powder, and it was found that the powder was firmly adsorbed on the surface of the needle tip [19], as shown in Fig. 4(c). Through investigation and research, it was found that the adhesion force between the needle tip and the powder was related to the environmental humidity, and the adhesion was significantly increased with an increase in the ambient humidity. Because the humidity was higher, the water molecules in the surrounding environment were more likely to condense between the contact surfaces, forming a meniscus liquid bridge and resulting in a more significant capillary force [20]. In order to reduce the influence of ambient humidity, the adhesion between the needle tip and the minute powder could be reduced by heating. The heating device was turned on, the heating probe was rapidly raised to $400{ }^{\circ} \mathrm{C}$, and the tungsten steel probe was heated by the heating device. At this time, the adhesion between the needle tip and the powder was greatly reduced, as shown in Fig. 4(d), and the needle tip surface was mostly free of the presence of powder.

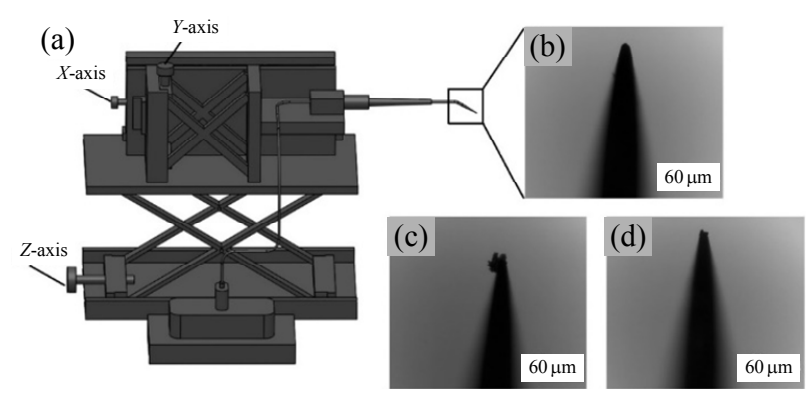

Fig. 4 3D moving heating micromanipulator diagram: (a) micromanipulator device moving in $X, Y$, and $Z$ 3D space, (b) magnified view of tungsten steel tip, (c) tungsten steel probe tip and fluorescent powder sticking attached together due to the presence of adhesion force, and (d) the reduced adhesion of the tip and the fluorescent powder when heated to $400{ }^{\circ} \mathrm{C}$.

\section{Experimental results and analysis}

\subsection{Assembly of fluorescent powder and microstructure}

The fluorescent powder was placed in a screen with a size of 180 meshes. The fluorescent powder having a particle diameter of fewer than $80 \mu \mathrm{m}$ was screened out and then transferred to a screen having a size of 200 meshes for screening, and the sieved particle diameter was less than $75 \mu \mathrm{m}$. The fluorescent powder with a particle diameter of 180 meshes to 200 meshes was retained and transferred to a clean glass slide. The "arch bridge" shaped microstructure processed on the glass substrate was fixed on the carrier platform of the 
transmission microscope, and the 3D micro-manipulation device was used to control the needle tip to pick up the fluorescent powder. The heating device was connected to the power supply, the probe was heated up to $400{ }^{\circ} \mathrm{C}$ for about $10 \mathrm{~s}$, and the temperature was transmitted to the needle tip. As the temperature of the needle tip increased, the adhesion force between the needle tip and the powder was greatly reduced, so that the fluorescent powder fell off from the needle tip. By using this method, the fluorescent powder was moved from the slide to the front of the microstructure, as shown in Fig. 5(a). The 3D micro-manipulation device was adjusted so that the needle tip touches the surface of the substrate and could be in contact with the fluorescent powder, as shown in Fig. 5(b). By adjusting the forward button of the 3D micro-manipulation device, the fluorescent powder was pushed by the tip of the needle towards the "arch bridge" shaped microstructure, until the
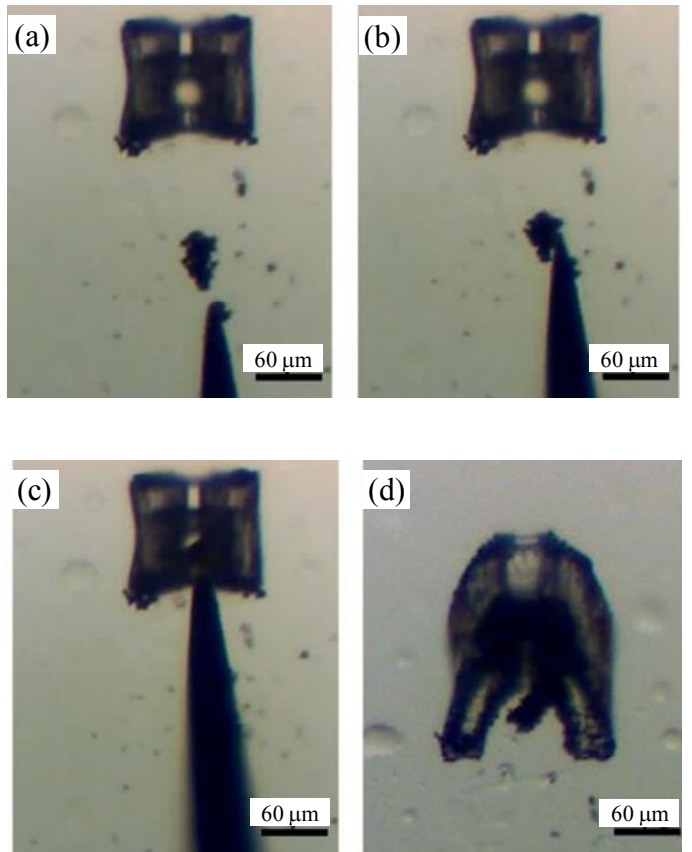

Fig. 5 Flow chart of fluorescent powder and "arch bridge" shaped microstructure micro-assembly: (a) placing the fluorescent powder in front of the "arch bridge" shaped microstructure with an unheated probe, (b) heating the probe to $400{ }^{\circ} \mathrm{C}$ and moving the fluorescent powder, (c) the fluorescent powder is assembled into the microstructure on a $2 \mathrm{D}$ plane with the heated probe, and (d) the assembled microstructure is tilted at an angle of $30^{\circ}$, and the fluorescent powder is placed in the center of the microstructure. powder was moved into the interior of the microstructure. Finally, the fluorescent powder entered the interior of the microstructure, as shown in Fig. 5(c). The assembled "arch bridge" shaped microstructure was observed at an angle of 30 degrees. It was found that the fluorescent powder is firmly fixed in the microstructure, because the glass substrate had a certain humidity, which led to the friction and electrostatic force between the fluorescent powder and the substrate, and the powder and the substrate could be bonded well.

\subsection{Fluorescence spectrum characterization}

In order to investigate whether the existence of the microstructure affects the performance of the fluorescent powder, the fluorescent powder in microstructures and pure fluorescent powder were respectively characterized by fluorescence spectroscopy [22], and the results are shown in Fig. 6. The microstructures assembled with fluorescent powder were placed under excitation light at $405 \mathrm{~nm}$ wavelength. Because the top of the microstructure has a circular hole with a diameter of $30 \mu \mathrm{m}$, the excitation light can be directly irradiated on the fluorescent powder through the circular hole. The emission wavelength of the fluorescent powder was $540 \mathrm{~nm}$, and the emission spectrum is shown as Curve B in Fig. 6. The fluorescent powder sieved through the 180 meshes and 200 meshes screens was placed on a glass slide and excited by excitation light at a wavelength of $405 \mathrm{~nm}$. The wavelength of the emitted light was also $540 \mathrm{~nm}$, and the obtained emission spectrum is shown as Curve A in Fig. 6. By comparing the two emission spectra of $\mathrm{A}$ and $\mathrm{B}$, we could observe that the peaks of the emission spectra of the fluorescent powder in microstructures and pure fluorescent powder were substantially coincided with each other, and the properties of the assembled fluorescent powder were not changed. This experiment has a certain reference value for fluorescent labeling and biological imaging experiments [23]. 


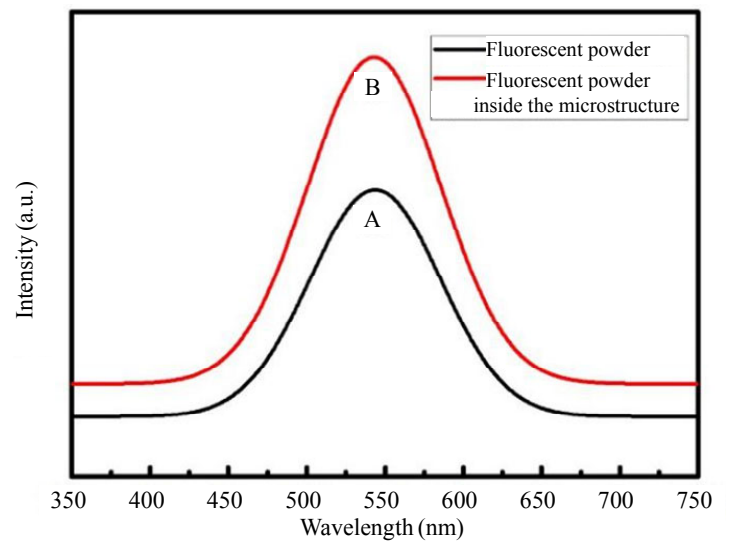

Fig. 6 Fluorescence spectrum of pure fluorescent powder and assembled fluorescent powder.

\subsection{Assembly of graphene powder and microstructure}

In order to verify that the laboratory-made $3 \mathrm{D}$ micro-operation heating device can assemble different objects into the microstructure, we next chose to assemble the graphene powder into the "arch bridge" shaped microstructure. Water-soluble graphene was extracted from the test tube, and deionized water was added and sonicated for hours, so that it was completely dissolved in deionized water. The graphene aqueous solution was taken in a $1.5 \mathrm{~mL}$ test tube, which was centrifuged in a centrifuge at a speed of $6000 \mathrm{r} / \mathrm{min}$ for $5 \mathrm{~min}$. The supernatant was taken and centrifuged again until there were no graphene particles at the bottom of the test tube. The cleaned slide on a heating platform was taken at $120^{\circ} \mathrm{C}$, the graphene supernatant was dropped in the center of the slide, and the supernatant was added again after deionized water was evaporated until a layer of graphene was deposited on the carrier plate. Thus, the graphene powder with a smaller particle size was obtained. The "arch bridge" shaped microstructure, made on the silicon substrate, was placed on the sample table of the transmission microscope and fixed by the sample clamp. In addition, to facilitate the observation, a light emitting diode (LED) light source as a light source was installed on the lens of the transmission microscope (because the silicon substrate was opaque, the light source at the bottom of the transmission microscope could not penetrate the silicon substrate). Then the graphene powder was stuck by the tip of the 3D micromanipulator without power supply and transferred to the front of the microstructure. At this time, the tip of the needle would reach $400{ }^{\circ} \mathrm{C}$ when the power supply of the micromanipulator was connected, which could be observed that graphene powder fell off from the tip about $100 \mu \mathrm{m}$ away from the microstructure, as shown in Fig. 7(a). The heated probe was brought into contact with the graphene powder, and since there was substantially no water vapor on the contact surface, the adhesion between the graphene powder and the probe tip was greatly reduced, as shown in Fig. 7(b). Adjusting the forward knob of the $3 \mathrm{D}$ moving device, the needle tip pushed the graphene powder into the interior of the microstructure smoothly, thereby successfully assembling the graphene powder into the "arch bridge" shaped microstructure, as shown in Fig.7(c).
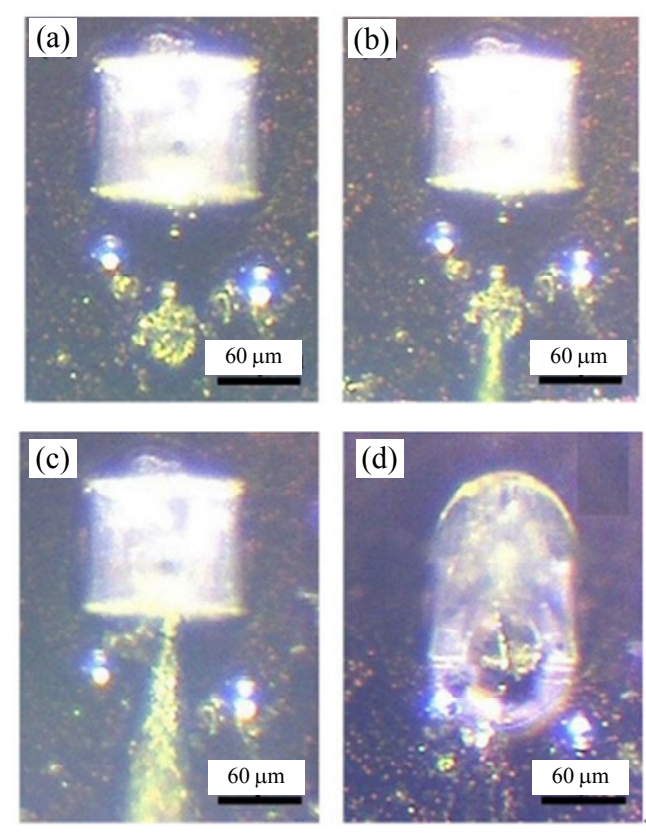

Fig. 7 Flow chart of graphene powder and "arch bridge" microstructure micro-assembly: (a) placing the graphene powder in front of the "arch bridge" shaped microstructure with an unheated probe tip, (b) heating the probe to $400{ }^{\circ} \mathrm{C}$ and moving the graphene powder, (c) using the heated probe to assemble the graphene powder into the microstructure on a $2 \mathrm{D}$ plane, and (d) observing the assembled microstructure at an inclined angle of $30^{\circ}$, the graphene powder was placed inside the microstructure. 
When the assembled "arch bridge" shaped microstructure was inclined at an angle of $30^{\circ}$, it was found that the graphene powder was firmly fixed in the interior of microstructure, as shown in Fig. 7(d).

\subsection{Graphene powder characterization}

Atomic force microscope (AFM) is a powerful tool to impart the crystallographic information of graphene powder including thickness and stacking order. Figure 8(a) displays the representative AFM images of the graphene powder after spin coating to a $300 \mathrm{~nm} \mathrm{SiO}_{2} / \mathrm{Si}$ substrate. The thicknesses of the graphene powder are approximately $0.5 \mathrm{~nm}-0.7 \mathrm{~nm}$, providing further evidence of the monolayer structure, as shown in Fig. 8(b). According to the size-distribution histogram [Fig. 8(c)], the thicknesses of the graphene powder is estimated to be $0.6 \mathrm{~nm}$.

Raman spectroscopy is one of the techniques used to characterize the rapid, non-destructive, and high resolution of carbon materials. It provides a vast amount of information and is an effective means to describe the internal structure and crystallinity of carbon materials. As shown in Fig. 8(d), Raman spectroscopy (QEPro, Ocean Optics) is performed to characterize graphene powder and the graphene powder inside the microstructure was carried out by excitation light at $532 \mathrm{~nm}$ wavelength $[24,25]$. In the Raman spectrum, the D peak located at $1350 \mathrm{~cm}^{-1}$ is caused by the symmetric stretching vibration of the $\mathrm{sp}^{2}$ carbon atoms in the aromatic ring requiring a defect to activate, which is usually used to measure the disorder of the carbon material. The $\mathrm{G}$ peak located at $1580 \mathrm{~cm}^{-1}$ is caused by the tensile vibration between the $\mathrm{sp}^{2}$ carbon atoms, corresponding to the plane vibration of the $E_{2 g}$ optical phonon at the center of the Brillouin zone, which is the characteristic peak of the carbon $\mathrm{sp}^{2}$ structure and mainly reflects the degree of ordering inside the carbonaceous material. The $2 \mathrm{D}$ peak located at near $2700 \mathrm{~cm}^{-1}$ originates from two phonon double

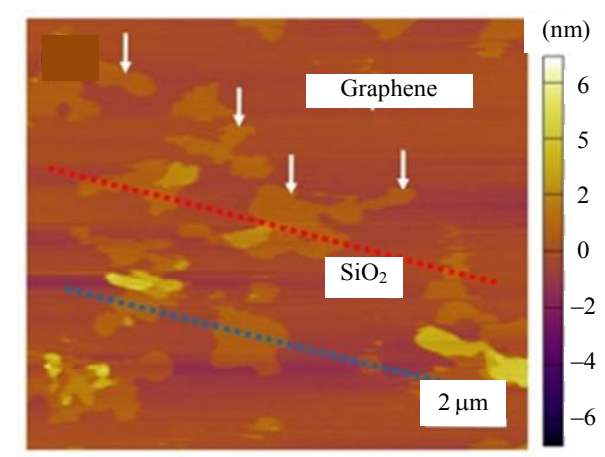

(a)

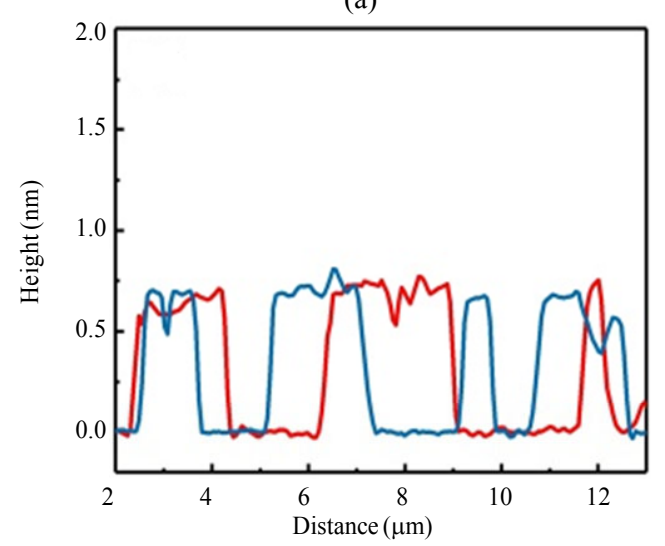

(b)

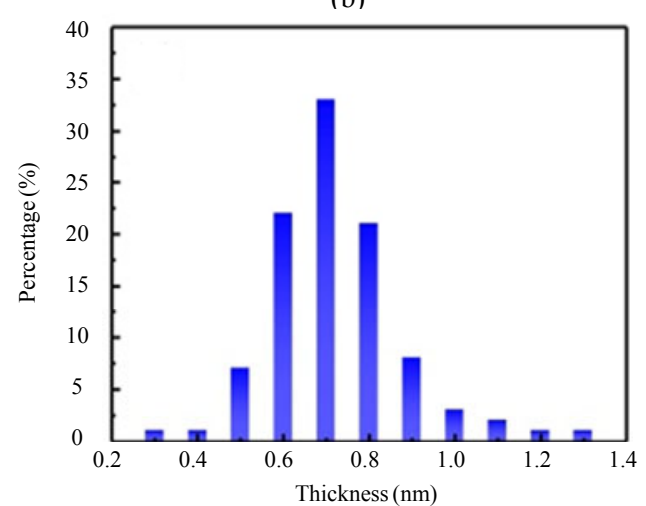

(c)

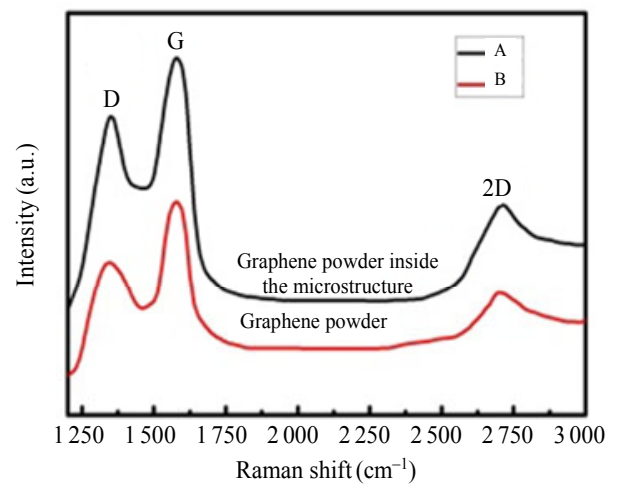

(d)

Fig. 8 Graphene powder characterization: (a) AFM images of graphene powder, (b) thickness profile along the line in (a), (c) flake thickness distribution of graphene powder, and (d) graphene and assembled graphene Raman spectroscopy. 
resonance transitions with opposite momentum in the carbon atoms, which is the unique peak position of graphene material [26]. The pure graphene powder is as shown by Curve B and the graphene powder in the microstructure is as shown by Curve A. The peak positions of the D peak, the G peak, and the $2 \mathrm{D}$ peak are basically the same, so the microstructure on the outside of the graphene material does not have any influence on its properties, and the graphene material and microstructure can be assembled.

\section{Conclusions}

In view of the fine 3D manufacture capability of the femtosecond laser, the "arch bridge" microstructure was firstly fabricated on glass and silicon slice substrates by 2PP. A self-made three-dimensional micromanipulation heating device was used to dip in the fluorescent powder and released at the fixed point, and then the fluorescent powder was accurately assembled into the interior of the microstructure. The excitation light of $405 \mathrm{~nm}$ wavelength passed through the circular hole at the top of the microstructure to directly irradiate the fluorescent powder in the microstructure. The emission spectrum of the fluorescent powder in the microstructure was substantially the same as that of the pure fluorescent powder, so the presence of microstructure had substantially no effect on the properties of the fluorescent powder. By using this assembly method, we tried to assemble graphene powder into microstructure. It was confirmed by experiments that the graphene powder could be easily assembled into the microstructure by the device. After Raman characterization, the properties of the graphene in the microstructure were not affected, so in this paper, a laboratory-made 3D micro-assembly device could be used to assemble a lot of micro-nano-sized powdery substances into the microstructure or to assemble different shapes of microstructures in a plane to form a hybrid microstructure. Because the micro-assembly device used in this article is manual, there are some limitations in accuracy. Later work begun to assemble an electric micro-assembly system by using a stepper motor to drive the micro-processing table to move, and used software to control, which could increase the operating accuracy. At the same time, we plan to use 2PP to fabricate microstructures and micro-needle under an oil immersion lens to reduce the size and improve the fineness of the microstructures. The same method can also be used to prepare micro-needle, so as to solve the problem of thicker needle tip.

\section{Acknowledgment}

We are grateful for the financial support from the National Natural Science Foundation of China (Grant Nos. 11704204 and 61604084) and the K. C. Wong Magna Fund in Ningbo University, China.

Open Access This article is distributed under the terms of the Creative Commons Attribution 4.0 International License (http://creativecommons.org/licenses/by/4.0/), which permits unrestricted use, distribution, and reproduction in any medium, provided you give appropriate credit to the original author(s) and the source, provide a link to the Creative Commons license, and indicate if changes were made.

\section{References}

[1] Y. L. Chen, F. Zhang, Q. Shan, and X. H. Zhang, "Application progress of precision forming technology in aerospace," Material Science and Technology, 2013, 21(4): 57-64.

[2] M. Mielke, "The hole story: Femtosecond manufacturing improves automobile fuel efficiency," Laser Focus World, 2013, 49(11): 35-41.

[3] L. D. Garza-García, L. M. Carrillo-Cocom, D. Araiz-Hernández, P. Soto-Vázquez, J. López-Meza, E. J. Tapia-Mejía, et al., "A biopharmaceutical plant on a chip: continuous micro-devices for the production of monoclonal antibodies," Lab on A Chip, 2013, 13(7): 1243.

[4] M. Khan, S. Mao, W. Li, and J. M. Lin, "Microfluidic devices in the fast-growing domain of single-cell analysis," Chemistry - A European 
Journal, 2018, 24(58): 15398-15420.

[5] G. Yang, J. A. Gaines, and B. J. Nelson, "A flexible experimental workcell for efficient and reliable wafer-level 3D micro-assembly," in Proceedings of 2001 ICRA. IEEE International Conference on Robotics and Automation (Cat. No.01CH37164), South Korea, May 21-26, 2001, pp. 133-138.

[6] J. Köhler, S. I. Ksouri, C. Esen, and A. Ostendorf, "Optical screw-wrench for microassembly," Microsystems and Nanoengineering, 2017, 3(1): 16083.

[7] M. J. Villangca, D. Palima, A. R. Bañas, and J. Glückstad, "Light-driven micro-tool equipped with a syringe function," Light: Science \& Applications, 2016, 5(9): e16148.

[8] A. B. Frazier, "Uses of polyimide for micromachining applications," in Proceedings of IECON'94 - 20th Annual Conference of IEEE Industrial Electronics, Italy, Sept. 5-9, 1994, pp. 1483-1487.

[9] K. Obata, A. EI-Tamer, L. Koch, U. Hinze, and B. N. Chichkov, "High-aspect 3D two-photon polymerization structuring with widened objective working range (WOW-2PP)," Light: Science \& Applications, 2013, 2(12): e116.

[10] U. Staudinger, G. Zyla, B. Krause, A. Janke, D. Fischer, C. Esen, et al., "Development of electrically conductive microstructures based on polymer/CNT nanocomposites via two-photon polymerization," Microelectronic Engineering, 2017, 179: 48-55.

[11] Q. Guo, R. Ghadiri, T. Weigel, A. Aumann, E. L. Gurevich, C. Esen, et al., "Comparison of in situ and ex situ methods for synthesis of two-photon polymerization polymer nanocomposites," Polymers, 2014, 6(7): 2037-2050.

[12] K. Zhang, K. Yang, S. Ai, and J. Xu, "Synthesis of novel cross-linked s-triazine-containing poly (aryl ether)s nanoparticles for biological fluorescent labeling," Designed Monomers and Polymers, 2017, 20(1): 389-396.

[13] D. Chu, X. Sun, Y. Hu, X. Dong, K. Yin, Z. Luo, et al., "Micro-channel etching characteristics enhancement by femtosecond laser processing high-temperature lattice in fused silica glass," Chinese Optics Letters, 2017, 15(7): 071403.

[14] B. Xu, W. Q. Du, J. W. Li, Y. L. Hu, L. Yang, C. C. Zhang, et al., "High efficiency integration of three-dimensional functional microdevices inside a microfluidic chip by using femtosecond laser multifoci parallel microfabrication," Scientific Reports, 2016, 6: 19989.
[15] A. Ostendorf and B. N. Chichkov, "Two-photon polymerization: a new approach to micromachining," Photonics Spectra, 2006, 40(10): 72.

[16] W. Xiong, Y. Liu , L. J. Jiang, Y. S. Zhou, D. W. Li, L. Jiang, et al., "Laser-directed assembly of aligned carbon nanotubes in three dimensions for multifunctional device fabrication," Advanced Materials, 2016, 28(10): 2002-2009.

[17] S. You, J. Li, W. Zhu, C. Yu, D. Mei, and S. Chen, "Nanoscale 3D printing of hydrogels for cellular tissue engineering," Journal of Materials Chemistry $B, 2018,6(15): 2187-2197$.

[18] A. Oláh, H. Hillborg, and G. J. Vancso, "Hydrophobic recovery of UV/ozone treated poly (dimethylsiloxane): adhesion studies by contact mechanics and mechanism of surface modification," Applied Surface Science, 2005, 239(3-4): 410-423.

[19] N. Dechev, W. L. Cleghorn, and J. K. Mills, "Microassembly of 3-D microstructures using a compliant, passive microgripper," Journal of Microelectromechanical Systems, 2004, 13(2): 176-189.

[20] M. Gauthier, N. Chaillet, P. Régnier, and N. Chaillet, "Analysis of forces for micromanipulations in dry and liquid media," Journal of Micromechatronics, 2008, 3(3): 389-413.

[21] Y. Ando, "The effect of relative humidity on friction and pull-off forces measured on submicron-size asperity arrays," Wear, 2000, 238(1): 12-19.

[22] Z. Song, F. Quan, Y. Xu, M. Liu, L. Cui, and J. Liu, "Multifunctional N, S co-doped carbon quantum dots with $\mathrm{pH}$-and thermo-dependent switchable fluorescent properties and highly selective detection of glutathione," Carbon, 2016, 104: 169-178.

[23] C. Wu and D. T. Chiu, "Highly fluorescent semiconducting polymer dots for biology and medicine," Angewandte Chemie International Edition, 2013, 52(11): 3086-3109.

[24] M. S. Dresselhaus, A. Jorio, M. Hofmann, G. Dresselhaus, and R. Saito, "Perspectives on carbon nanotubes and graphene Raman spectroscopy," Nano Letters, 2010, 10(3): 751-758.

[25] T. Kaplas, A. Matikainen, T. Nuutinen, S. Suvanto, P. Vahimaa, and Y. Svirko, "Scalable fabrication of the graphitic substrates for graphene-enhanced Raman spectroscopy," Scientific Reports, 2017, 7(1): 8561.

[26] W. Xiong, Y. S. Zhou, W. J. Hou, L. J. Jiang, Y. Gao, L. S. Fan, et al., "Direct writing of graphene patterns on insulating substrates under ambient conditions," Scientific Reports, 2014, 4(7499): 4892. 